\title{
Disentangling density and temperature effects in the viscous slowing down of glassforming liquids
}

\author{
G. Tarjus,${ }^{1}$ D. Kivelson ${ }^{2}$ S. Mossa,${ }^{1}$ and C. Alba-Simionesco ${ }^{3}$ \\ ${ }^{1}$ Laboratoire de Physique Théorique des Liquides, \\ Université Pierre et Marie Curie, 4 place Jussieu, Paris 75005, France \\ ${ }^{2}$ Department of Chemistry and Biochemistry, University of California, Los Angeles, California 90095 \\ ${ }^{3}$ Laboratoire de Chimie Physique, Bâtiment 349, \\ Université de Paris Sud, F-91405 Orsay, France
}

(Dated: November 20, 2018)

\begin{abstract}
We present a consistent picture of the respective role of density $(\rho)$ and temperature $(T)$ in the viscous slowing down of glassforming liquids and polymers. Specifically, based in part upon a new analysis of simulation and experimental data on liquid ortho-terphenyl, we conclude that a zeroth-order description of the approach to the glass transition should be formulated in terms of a temperature-driven super-Arrhenius activated behavior rather than a density-driven congestion or jamming phenomenon. The density plays a role at a quantitative level, but its effect on the viscosity and the $\alpha$-relaxation time can be simply described via a single parameter, an effective interaction energy that is characteristic of the high- $T$ liquid regime; as a result, $\rho$ does not affect the "fragility" of the glassforming system.
\end{abstract}

\section{INTRODUCTION}

Why should one care about the respective role of the various external control parameters, temperature $T$, pressure $P$, density $\rho$ (or volume $V$ ) in the viscous slowing down of glass forming liquids and polymers? If the glass transition at $T_{g}$ were a bona fide critical point with a diverging correlation length, the question of how it is approached in the $P-T$ or $\rho-T$ phase diagram would be interesting but not crucial to determine the main features of the critical slowing down. However, there is no directly observed critical point associated with the glass transition of liquids and polymers. For the canonical fragile glassformer ortho-terphenyl, the often invoked "ideal glass transition temperature" obtained by extrapolating the Vogel-Fulcher-Tammann expression for the viscosity and the $\alpha$ (or primary) relaxation time to infinity or extrapolating the excess entropy to zero, is some $40 \mathrm{~K}$ below $T_{g}$ 1, 2]. Various theories predict "avoided critical points" at temperatures above $T_{g}$, such as the mode-coupling singularity at $T_{c}[\underline{3}$ ] or the frustrationlimited-domain avoided transition at $T^{*}[4]$, or "unreachable critical points" at temperatures below $T_{g}$, such as the ideal glass transition at $T_{o}$ [5, 6, 7], but whatever the approach, the putative correlation lengths are expected to remain of modest size, no more than 5 to 10 molecular diameters [1, 2]. The question of which control parameter dominates the slowing down of the relaxation may then be relevant to better understand the underlying physics.

Actually, most zeroth-order theoretical descriptions of the slow dynamics of glass forming liquids are formulated either at constant temperature or at constant density. Two such limiting approaches are:

1) the congestion, or jamming, picture of the glass transition, in which slowing down results from the drainage of free volume as density increases [8, 9]. The paradigm for this approach is the hard-sphere fluid;
2) a description in terms of thermally activated processes on a constant-density energy landscape [10, 11]. In this approach the landscape does not change as one cools the system ${ }^{1}$, and the super-Arrhenius $T$-dependence of the $\alpha$-relaxation time and the viscosity is attributed to changes in the minima and barriers encountered in the exploration of the landscape as one lowers the temperature.

Of course, such zeroth-order descriptions can always be improved to account for some additional dependence on the "secondary" control variable: temperature can be included in the hard-sphere picture by allowing the diameter of the spheres to change with temperature, and the energy landscape can be studied for various densities. But the mechanism responsible for the viscous slowing down is determined predominantly by the density in the former case and the temperature in the latter one.

In this article, we extend our previous work [12, 13] to develop a consistent picture of the roles of density and temperature. To do so, we consider recent studies made by others as well as new simulation and experimental data. The main conclusion is that the appropriate zerothorder description of the slowing down of relaxation as one approaches the glass transition is not that of density (or volume)-driven congestion or jamming, but that of a temperature-driven super-Arrhenius activated behavior. Density plays a role at a quantitative level: there is a significant density dependence of the $\alpha$-relaxation time and the viscosity at fixed temperature. However, the density dependence can be described in terms of a single interaction energy that is characteristic of the high-T Arrhenius

\footnotetext{
${ }^{1}$ For a given number of particles $N$ and a given volume $V$, hence a given density $\rho=N / V$, the potential energy landscape, i.e., the hypersurface of potential energy plotted as a function of the $3 N$ configurational coordinates, is independent of temperature.
} 
liquid. As a result, the temperature dependence of the $\alpha$-relaxation time and the viscosity at different densities collapse onto a single, material-dependent curve; most importantly, this implies that density does not affect the "fragility" of a glass former, i.e., the degree to which it exhibits super-Arrhenius temperature dependence.

\section{ASSESSING THE INFLUENCE OF DENSITY AND TEMPERATURE}

Whereas physical pictures and theories are conveniently formulated in terms of temperature and density, experiments are usually carried out by using temperature and pressure as control variables. Standard studies of the slowing down of relaxation leading to the glass transition are performed along isobars, typically at atmospheric pressure, by cooling the liquid or the polymer. The resulting $T$-dependence of, say, the $\alpha$-relaxation time $\tau_{\alpha}$ includes both an intrinsic temperature effect and a density effect resulting from the fact that cooling a system at constant pressure usually increases its density. The temperature typically decreases by a factor of 2 between the normal liquid and the glass transition, whereas in the same range the density increases typically by only $10 \%$; but of course those numbers are not meaningful by themselves, the important physical information being the sensitivity of the slowing down to changes of $T$ and $\rho$.

In order to disentangle the effects of temperature and density at constant pressure and provide a quantitative, model-free assessment of their relative importance, we suggested some years ago 12 to use as a criterion the ratio of two coefficients of expansivity, $\left|\alpha_{\tau}\right| / \alpha_{P}$, where $\alpha_{P}=-\rho^{-1}(\partial \rho / \partial T)_{P}$ is the usual isobaric expansivity and $\alpha_{\tau}=-\rho^{-1}(\partial \rho / \partial T)_{\tau}$ is the (unusual) isochronic expansivity for a constant $\alpha$-relaxation time or a constant viscosity $(\eta)$. Indeed, along an isobar the variation with $T$ of $\ln \left(\tau_{\alpha}\right)$ where $\tau_{\alpha}$ is expressed, say, in seconds (or $\ln (\eta)$ with $\eta$ expressed, say, in Poise) can be decomposed as

$$
\left.\frac{\partial \ln \left(\tau_{\alpha}\right)}{\partial T}\right|_{P}=\left.\frac{\partial \ln \left(\tau_{\alpha}\right)}{\partial T}\right|_{\rho}+\left.\left.\frac{\partial \ln \left(\tau_{\alpha}\right)}{\partial \rho}\right|_{T} \frac{\partial \rho}{\partial T}\right|_{P},
$$

where the first term of the right-hand side is the intrinsic effect of temperature at constant density and the second one is the effect of density at constant temperature. By using the above mentioned coefficients of expansivity, it is easy to show that the former term is expressed in terms of $\alpha_{\tau}$ and the latter in terms of $\alpha_{P}$, so that

$$
\left.\frac{\partial \ln \left(\tau_{\alpha}\right)}{\partial T}\right|_{P}=\left.\frac{\partial \ln \left(\tau_{\alpha}\right)}{\partial \ln (\rho)}\right|_{T}\left(-\alpha_{\tau}+\alpha_{P}\right)
$$

and the ratio $\left|\alpha_{\tau}\right| / \alpha_{P}$ measures the relative importance of temperature versus density contributions at a given thermodynamic point (recall that in general $\alpha_{P}>0$ and $\left.\alpha_{\tau}<0\right)$ : if $\left|\alpha_{\tau}\right| / \alpha_{P}>1$, temperature is a more important controlling factor of the viscous slowing down than density.

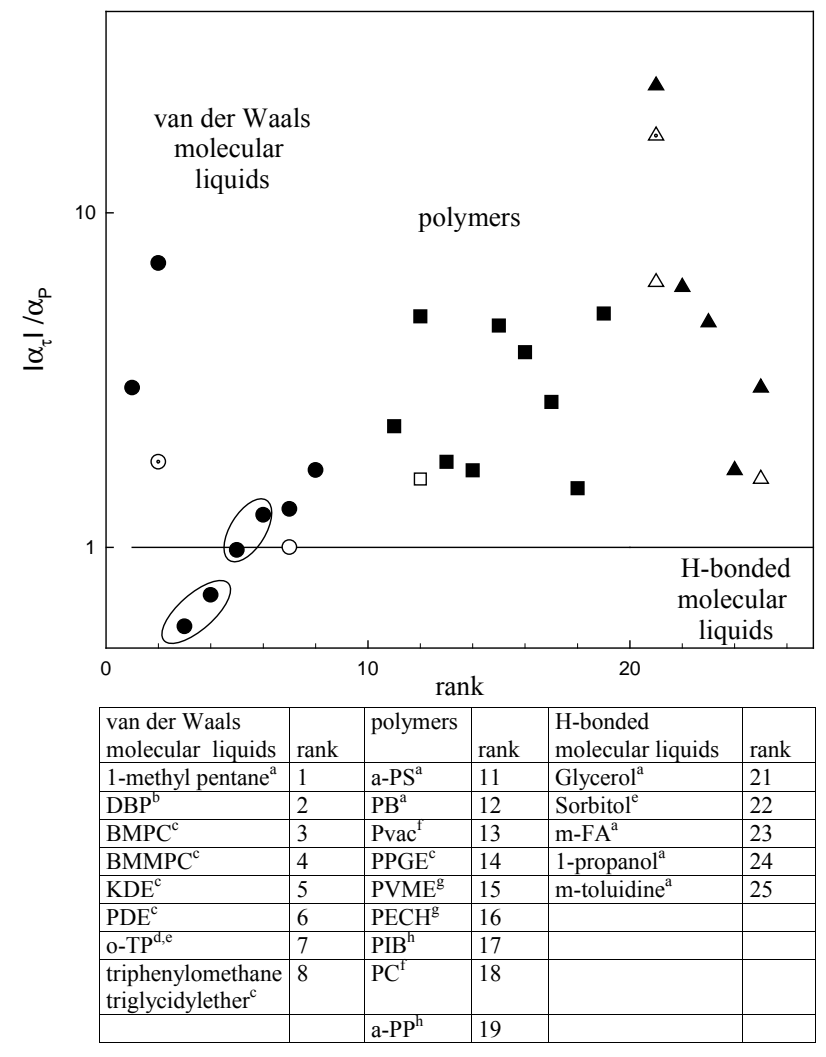

FIG. 1: Ratio $\left|\alpha_{\tau}\right| / \alpha_{P}$ for various liquids and polymers evaluated at the glass transition and at higher temperature (at atmospheric pressure): filled symbols for $T_{g}$ (see text), dotted open symbols for $\tau_{\alpha} \sim 10^{-5} \mathrm{~s}$ or $\tau_{\alpha} \sim 10^{-1} \mathrm{~s}$, open symbols for $\tau_{\alpha} \sim 10^{-9}$ s or $\eta \sim 10^{4}$ cPoise. The two ellipses indicate points corresponding to liquids of the same family with a very similar chemical structure. The point for DBP at Tg should be taken with caution because of the inaccuracy of the equation of state. The rank of the liquids (see the table) within the three classes, van der Waals molecular liquids, polymers, and H-bonded molecular liquids, is arbitrarily chosen. References for the table: (a) Ref. [12] and references therein, (b) Refs. [27], [28] and [18], (c) Ref. [15], (d) Ref. [23], (e) Ref. [1], (f) Ref. [31], (g) Ref. [29], (h) Ref. [30], (i) Ref. 32].

Note that an equivalent characterization of the respective weights of temperature and density, although not expressed in terms of thermodynamic coefficients of expansivity, was proposed by Naoki et al. [14]. These authors suggested use of the ratio $E_{V} / H_{P}$, where $E_{V}=$ $\left(\partial \ln \left(\tau_{\alpha}\right) / \partial(1 / T)\right)_{\rho}$ and $H_{P}=\left(\partial \ln \left(\tau_{\alpha}\right) / \partial(1 / T)\right)_{P}$. It is easy to show that

$$
\frac{E_{V}}{H_{P}}=\frac{\left|\alpha_{\tau}\right|}{\left|\alpha_{\tau}\right|+\alpha_{P}}
$$

so that, when $\left|\alpha_{\tau}\right| / \alpha_{P}>1, E_{V} / H_{P}>1 / 2$.

From available experimental data on viscosity and $\alpha$ relaxation time we have calculated (or collected) the ratio $\left|\alpha_{\tau}\right| / \alpha_{P}$ for a variety of glassforming liquids and polymers interacting through van der Waals forces or hydrogen bonds. Strong, network-forming systems are not con- 
sidered here ${ }^{2}$. We show in Fig. 1 1 the ratios calculated at atmospheric pressure (i) at $T_{g}$ (either the calorimetric $T_{g}$ corresponding to an isochrone with $\tau_{\alpha} \sim 1000 \mathrm{~s}$ or the dielectric $T_{g}$ with $\tau_{\alpha} \sim 1-100 \mathrm{~s}$ ) and (ii), when possible, for a smaller $\alpha$-relaxation time or viscosity (i.e., a higher temperature). It is quite noticeable that the ratio at $T_{g}$ is significantly larger than 1 , with the exception of a family of organic liquids with very similar chemical structure (BMPC, BMMPC) for which it is slightly less than 1 [15]. The largest value is for the $H$-bonded liquid glycerol; van der Waals molecular liquids tend to have smaller ratios than polymers and $\mathrm{H}$-bonded liquids, but the correlation between the value of the ratio and the nature of the glassforming system is not very strong.

A few additional comments are worth making. First, there may be significant errors in determining $\alpha_{\tau}$ and $\alpha_{P}$ : high-precision thermodynamic and dynamic data are needed to obtain such second-order quantities. Secondly, and maybe somehow contrary to the expectation that density becomes more important as pressure increases, the ratio $\left|\alpha_{\tau}\right| / \alpha_{P}$ is found to increase with pressure (except for glycerol), at least up to a few kbars. Finally, the ratio decreases as the temperature increases or, equivalently, as $\tau_{\alpha}$ (or $\eta$ ) decreases. It is indeed expected that in the "normal", high- $T$ liquid density and temperature play more comparable roles, at least for weakly interacting systems. However, this is rather at odds with the congestion/jamming picture of the glass transition in which one would expect density to become predominant as one gets close to $T_{g}$.

To summarize the results of this section: overall, temperature dominates over density in driving the viscous slowing down of glassforming liquids and polymers; for a quantitative description one cannot bluntly neglect the effect of density; the role of temperature becomes more important in the super-Arrhenius regime as one approaches the glass transition.

\section{SCALING OUT THE DENSITY DEPENDENCE}

The conclusions of the preceding section, together with previous work [12, 13], lead us to describe the $\alpha$ relaxation in supercooled liquids and in polymers as thermally activated, i.e., for practical purposes describable by the following $T$-dependence:

$$
\tau_{\alpha}(\rho, T)=\tau_{\alpha}^{\infty}(\rho) \exp [E(\rho, T) / T],
$$

where the effective activation energy $E(\rho, T)$ is at least equal to several times the thermal energy $T$ (we work in

\footnotetext{
${ }^{2}$ It is likely that for such strongly interacting glassformers temperature is the dominant driving parameter of the viscous slowing down so long as pressure does not change the local structure of the liquid.
}

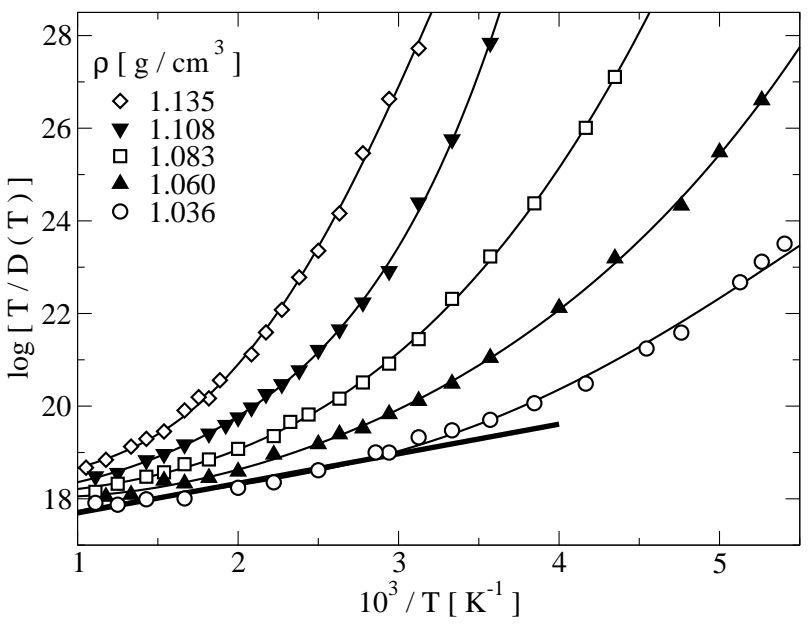

FIG. 2: Simulation data on a model ortho-terphenyl: $\ln (T / D(\rho, T))$, where $D(\rho, T))$ is the translational diffusion constant, versus $1 / T$ for several values of $\rho$ (from top to bottom, $\left.\rho=1.135,1.108,1.083,1.060,1.036 \mathrm{~g} / \mathrm{cm}^{3}\right)$. The straight line is an Arrhenius fit to the high-temperature portion of the data $\left(T \leq T^{*}(\rho)\right)$, shown here for $\rho=1.036 \mathrm{~g} / \mathrm{cm}^{3}$.

units such that $k_{B}=1$ ). A zeroth-order physical picture of the viscous slowing down of fragile glass formers and of their crossover from Arrhenius to super-Arrhenius behavior should thus focus on the role of temperature, the effect of density being incorporated in quantities characterizing the high- $T$, "normal" liquid phase. As we suggested in Ref. 13], the simplest description is that density only enters via a characteristic interaction energy that one can associate with the energy barrier to $\alpha$-relaxation and viscous flow in the high- $T$ Arrhenius-like regime, $E_{\infty}(\rho)$; this energy sets the scale for both the effective activation energy $E(\rho, T)$ and the temperature. Accordingly, all isochoric data should collapse onto a master curve

$$
\frac{E(\rho, T)}{E_{\infty}(\rho)}=\Phi\left(\frac{T}{E_{\infty}(\rho)}\right)
$$

where $\Phi(\zeta)$ is a monotonously decreasing function of $\zeta$ that is equal to 1 for $\zeta$ larger than some crossover value $\zeta^{*}$. If, moreover, the $\rho$-dependence of the prefactor $\tau_{\alpha}^{\infty}$ in Eq. (4) is negligible, one can also obtain a mastercurve for the $\alpha$-relaxation time itself,

$$
\ln \left(\tau_{\alpha}(\rho, T)\right)=\ln \left(\tau_{\alpha}^{\infty}\right)+\frac{E_{\infty}(\rho)}{T} \Phi\left(\frac{T}{E_{\infty}(\rho)}\right),
$$

where $\tau_{\alpha}(\rho, T)$ and $\tau_{\alpha}^{\infty}$ are both expressed, say, in seconds. It is clear from Eqs. (5) and (6) that the effect of density could be quantitatively significant without qualitatively determining the slowing down of the relaxation as one approaches the glass transition.

In Ref. 13] we gave two examples of data collapse onto the mastercurve in Eq. (515) (for the simulation data on the binary Lennard-Jones mixture [16] and for experimental data on glycerol 17, 18]). It is worth stressing that, despite decades of pressure studies of glassforming liquids 


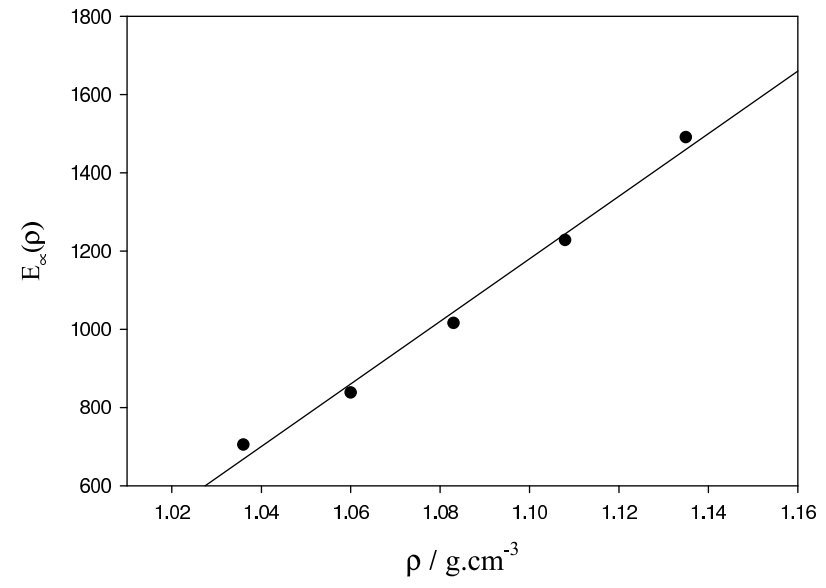

FIG. 3: High- $T$ Arrhenius activation energy $E_{\infty}$ versus density for the data shown in Fig. 2

and polymers, the available data base of isochoric data is scant. In what follows we present new simulation data on a model ortho-terphenyl and, building upon the work of Refs. [19] and 20] we also analyze experimental data of ortho-terphenyl.

\section{ANALYSIS OF SIMULATION AND EXPERIMENTAL DATA ON ORTHO-TERPHENYL}

We have considered the simulation data of Ref. 21] for the now widely studied Lewis-Wahnström model of ortho-terphenyl 22], a model of rigid three-site molecules with intermolecular site-site Lennard-Jones interactions. In view of the analysis below, the data base of state points has been further extended in the high-temperature region. Simulation details are given in Ref. 21]. The translational diffusion constant $D(\rho, T)$ has been considered for 5 different densities in the range 1.036 to 1.135 $\mathrm{g} / \mathrm{cm}^{3}$ (representing a $10 \%$ overall change in density) ${ }^{3}$. To obtain data more akin to viscosity or relaxation time, we have considered $T / D(\rho, T)$ : see Fig. 2 From the Arrhenius plot of Fig. 2] we have determined for each curve a high- $T$ activation energy $E_{\infty}(\rho)$ that describes the data above some crossover temperature $T^{*}(\rho)$, below which the curves markedly deviate from an Arrhenius dependence. The values of $E_{\infty}(\rho)$ are shown in Fig. 3. As can be seen from Fig. 4 the data taken along the 5 different isochores collapse onto a single curve when energies and temperatures are rescaled by the parameter $E_{\infty}(\rho)$; the prefactor $\tau_{\alpha}^{\infty}$ being independent of density, a master curve is obtained by using either Eq. (5) (Fig. 4 Top) or

\footnotetext{
${ }^{3}$ Note that, in the range of temperature accessible to our computer simulations, there is no significant decoupling between the translational diffusion constant and the other relaxation times.
}
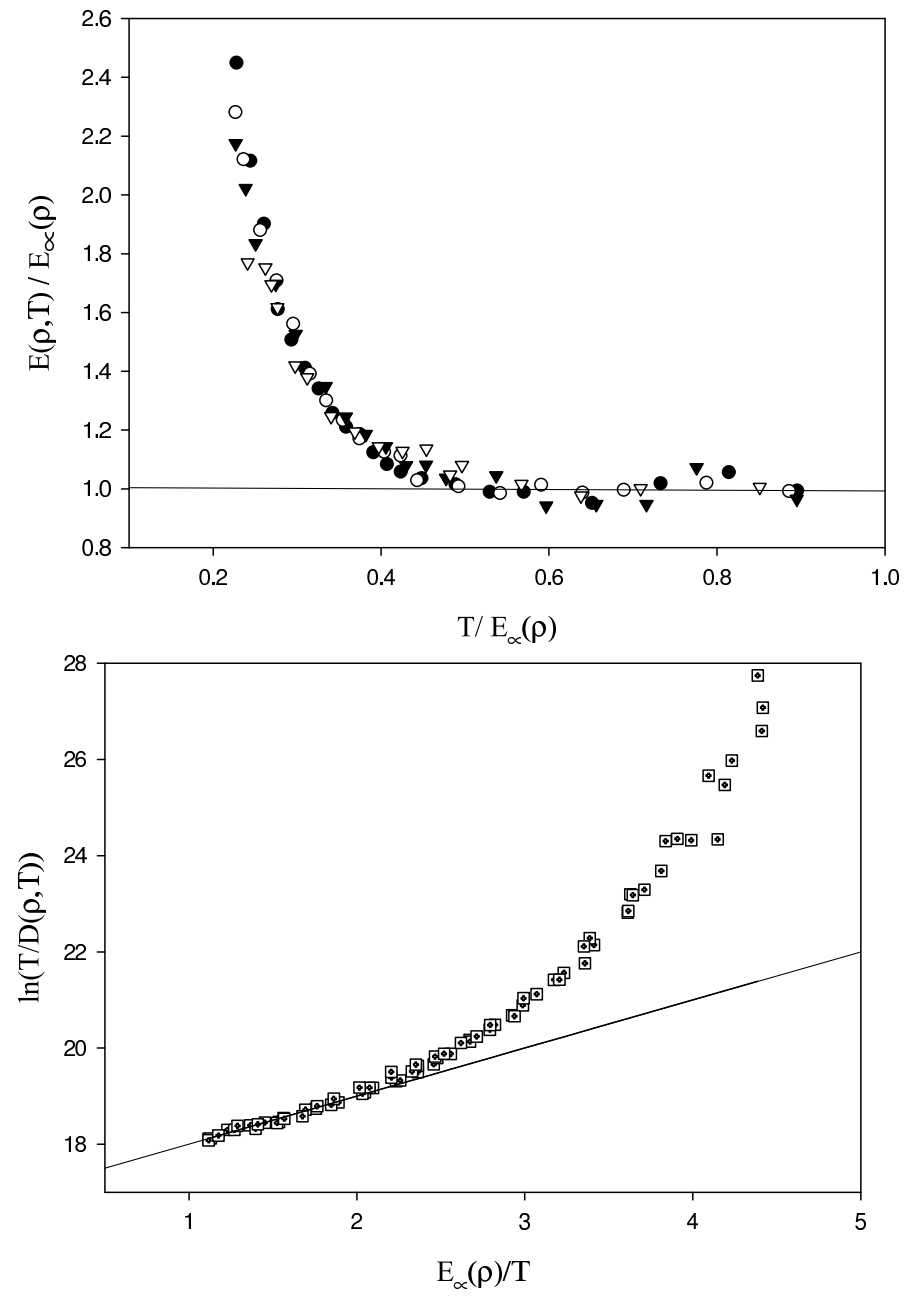

FIG. 4: Mastercurves for the simulation data on model orthoterphenyl at 5 different densities: Top: $E(\rho, T) / E_{\infty}(\rho)$ versus $T / E_{\infty}(\rho)$; Bottom: $\ln (T / D(\rho, T))$ versus $E_{\infty}(\rho) / T$. Symbols as in Fig. 2

\section{Eq. (6) (Fig. 团Bottom).}

Computer simulation offers a convenient means to study isochoric data directly, but the range of temperature and of relaxation time attainable is quite limited. Ideally, isochoric experimental data would provide a more stringent test for Eqs. (5) or (6). However, even for the well-studied liquid ortho-terphenyl, obtaining isochoric data for the viscosity or the $\alpha$-relaxation time requires significant manipulation of the original data (collected along isobars and isotherms). We follow here the work of others [20] who gave evidence that the $\alpha$-relaxation time of ortho-terphenyl for a large range of pressure and temperature, hence of density and temperature, can be placed to a good accuracy on a single master curve when it is plotted as a function of $\rho T^{-1 / 4}$ : see, e.g., Fig. 11 of Ref. 20]. We have repeated a similar analysis for the viscosity, extending the range to higher temperatures by using the data of Schug et al. 23]. The resulting mastercurve, $\ln (\eta)$ versus $\rho^{4} / T$ is shown in Fig. 15 (the inset 


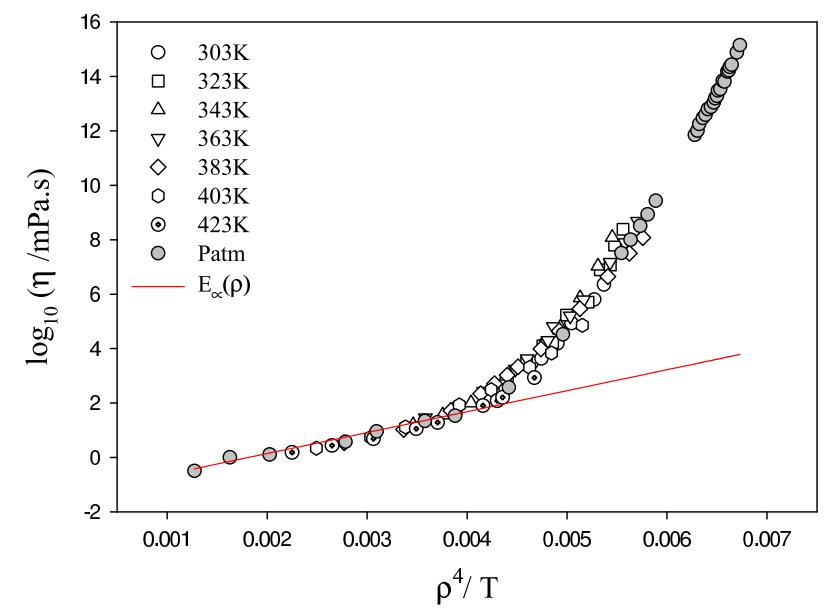

FIG. 5: Mastercurve experimental data on the viscosity of liquid ortho-terphenyl: $\log _{10}(\eta)$ versus $\rho^{4} / T$. The data at atmospheric pressure are taken from Refs. [33, 34] and those at higher pressures from Ref. [23] (only pressures up to 5 kbars are considered). The straight line is the best Arrhenius fit to the high- $T /$ low- $\rho$ portion of the data.

shows a similar plot for the $\alpha$-relaxation time). Data collapse is very good. One should however keep in mind a few words of caution. First, there is a significant discrepancy between $\alpha$-relaxation times obtained by different techniques. Secondly, we have included data only up to 5 kbars (similarly in Ref. 20] only data up to 2 kbars were considered); for higher pressures, the viscosity data start to deviate from the mastercurve, which may be due to a much more uncertain equation of state, to experimental errors, and/or to the fact that the $\rho T^{-1 / 4}$ scaling somewhat breaks down at very high pressures.

What is the meaning of the $\rho T^{-1 / 4}$ scaling? In Ref. 19] it was suggested that this scaling is reminiscent of that occurring in the fluid of soft spheres interacting via an $r^{-12}$ potential. Indeed for particles interacting via a power-law $r^{-n}$ pair potential, the $\rho$ and $T$ dependences of all excess thermodynamic properties ${ }^{4}$ enter only through a single parameter $\Gamma=\rho T^{-3 / n}[24]$; the relaxation time is then essentially a function of $\Gamma$ whereas the equation of state takes the form $P T^{-(1+3 / n)}=$ $\mathcal{F}\left(\rho T^{-3 / n}\right)$. It would be, however, somewhat surprising if a molecular liquid such as ortho-terphenyl behaved like a fluid of soft repulsive spheres (with $n=12$ as in a Lennard-Jones potential). Actually, from the data in the literature it is easy to check that the equation of state does not obey the predicted form $P T^{-5 / 4}=\mathcal{F}\left(\rho T^{-1 / 4}\right)$.

We suggest instead that the empirical scaling found for the viscosity and the $\alpha$-relaxation time of ortho-terphenyl

\footnotetext{
4 An excess thermodynamic quantity is defined here as the contribution due to the interactions that comes on top of an ideal-gas result.
}

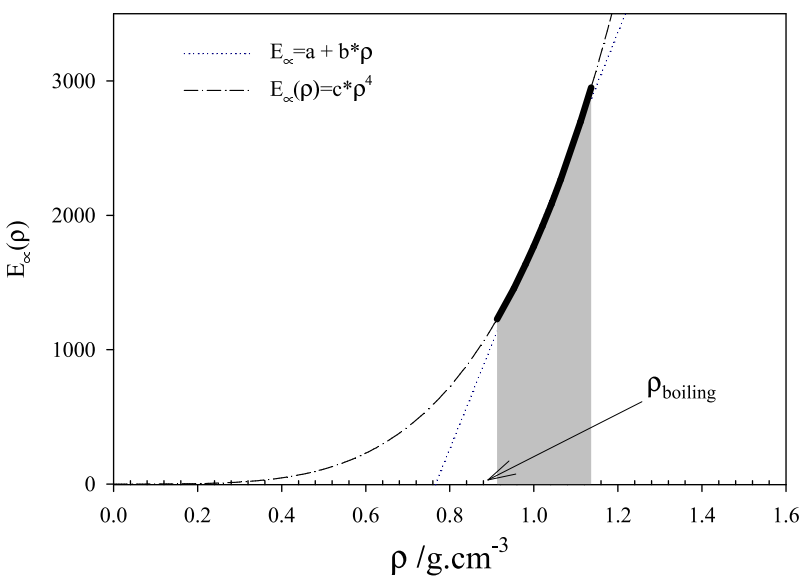

FIG. 6: High- $T$ Arrhenius activation energy $E_{\infty}$ versus density from the viscosity data of ortho-terphenyl. The dotdashed curve is the $\rho^{4}$-dependence derived from Fig. [5] the dotted line is a linear fit to the $\rho^{4}$-curve over the relevant range of liquid densities (shaded area).

is one more manifestation of the mastercurve discussed in the preceding section: the data collapse shown in Fig. 5 is a special case of Eq. (6) with $E_{\infty}(\rho) \propto \rho^{4}$. One can indeed see from Fig. 15 that the small- $(1 / \zeta)$ portion of the curve, where $1 / \zeta=\rho^{4} / T$, is linear to a good approximation, which corresponds to an Arrhenius behavior, $\ln (\eta(\rho, T))=\ln \left(\eta_{\infty}\right)+E_{\infty}(\rho) / T$, with $E_{\infty}(\rho)$ proportional to $\rho^{4}$. In Fig. [6] we have plotted $E_{\infty}(\rho)$ for the range of densities actually studied experimentally: it is obvious from the figure that for this range of densities, a linear fit to $E_{\infty}(\rho)$ would do just as well as the power law $\rho^{4}$. This latter may then just be a coincidence without any substantial physical meaning. On the other hand, the observed data collapse supports our claim that the influence of the density on the slow dynamics of glassformers can be reduced to a single effective interaction energy $E_{\infty}(\rho)$.

\section{COMPARISON BETWEEN SYSTEMS: RELATIVE "FRAGILITIES"}

The scaling function $\Phi(\zeta)$ appearing in the mastercurves given in Eqs. (5) and (6) is species-specific, as are the $\rho$-dependence and the magnitude of the effective interaction energy $E_{\infty}(\rho)$. This is illustrated in Figs. 7 and 8 for the two systems studied in the present work, computer simulated and real ortho-terphenyl, as well as for the two systems considered in a previous study, the binary Lennard-Jones mixture and (real) glycerol. In Fig. 17 we have plotted $E_{\infty}(\rho) / E_{\infty}\left(\rho_{\text {ref }}\right)$ versus $\rho / \rho_{\text {ref }}$, where $\rho_{\text {ref }}$ is a liquid density taken as a reference (e.g., the density at the boiling point when it is known), for the four glassformers. One observes that besides the fact that over the range considered (a variation of about 10\%) the $\rho$-dependence is essentially linear, the four curves are 


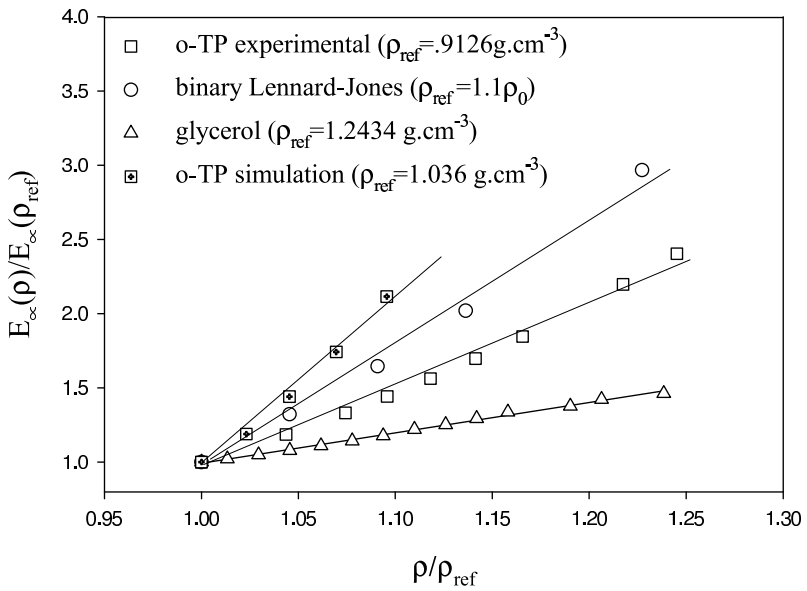

FIG. 7: $E_{\infty}(\rho) / E_{\infty}\left(\rho_{\text {ref }}\right)$, where $\rho_{\text {ref }}$ is a small reference liquid density, for the glassformers: binary Lennard-Jones mixture, glycerol, Lewis-Wahnström model of ortho-terphenyl (see Fig. 3), and ortho-terphenyl (see Fig. 6).

all different ${ }^{5}$.

In Fig. 8 we show the curves $E(\rho, T) / E_{\infty}(\rho)$ versus $\ln \left(T / E_{\infty}(\rho)\right)$ and $T / T^{*}(\rho)$, where $T^{*}(\rho)=\zeta_{*} E_{\infty}(\rho)$ is the crossover temperature below which a marked superArrhenius $T$-dependence is observed; in terms of mastercurves (see Eq. (5)), this amounts to plotting $\Phi(\zeta)$ versus $\zeta / \zeta_{*}$ where $\Phi(\zeta) \simeq 1$ for $\zeta \leq \zeta_{*}, \zeta_{*}$ being a speciesdependent constant. The differences observed for $\zeta>\zeta_{*}$ among the four systems reflect the different degrees of super-Arrhenius behavior, i.e., the different fragilities: one finds, as expected, that (real) ortho-terphenyl and glycerol are significantly more fragile than the two computer simulated systems (the highest points in activation energy for glycerol should be taken with caution [13]).

It is worth pointing out that a consequence of the existence of the mastercurves is that fragility is independent of density. This applies no matter what particular definition of (dynamic) fragility 25] one chooses. Consider, for instance, the commonly used steepness index,

$$
m_{\rho}=\left.\frac{\partial \ln \left(\tau_{\alpha}(\rho, T)\right)}{\partial\left(T_{g}(\rho) / T\right)}\right|_{T_{g}(\rho)},
$$

evaluated along an isochoric path. Applying Eq. (6) and defining as usual $T_{g}(\rho)$ as the temperature at which $\tau_{\alpha}(\rho, T)$, expressed in seconds, is equal to a given value, $\ln \left(\tau_{\alpha}(\rho, T)\right)=\mathcal{K}$, one finds that

$$
m_{\rho}=\left.\frac{E_{\infty}(\rho)}{T_{g}(\rho)} \frac{\partial \ln \left(\tau_{\alpha}(\rho, T)\right)}{\partial\left(T_{g}(\rho) / T\right)}\right|_{T_{g}(\rho)}=-\left.\zeta_{g} \frac{d(\Phi(\zeta) / \zeta)}{d \zeta}\right|_{\zeta_{g}}
$$

\footnotetext{
5 This, as well as the results in Fig. 8 casts some doubts on the ability of the Lewis-Wahnström model to accurately represent liquid ortho-terphenyl.
}

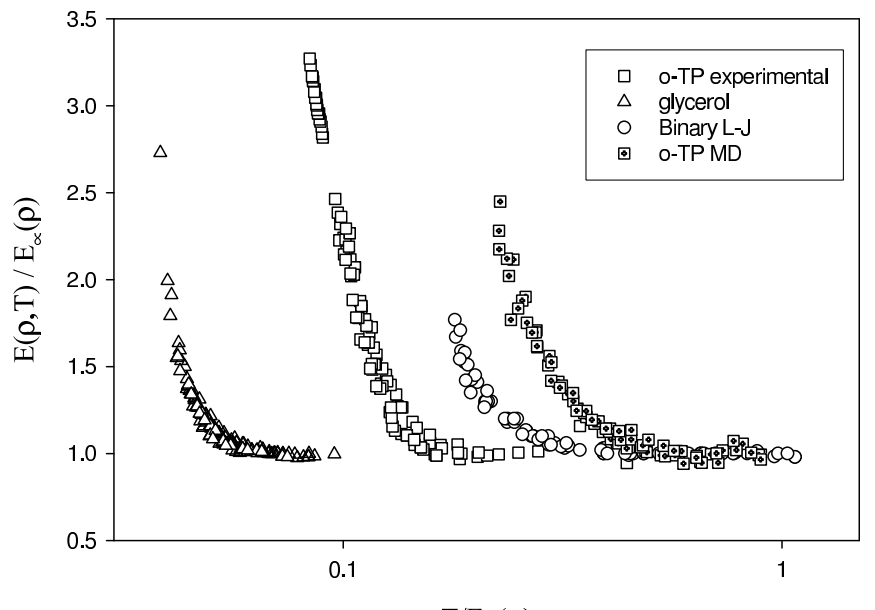

$\mathrm{T} / \mathrm{E}_{\propto}(\rho)$

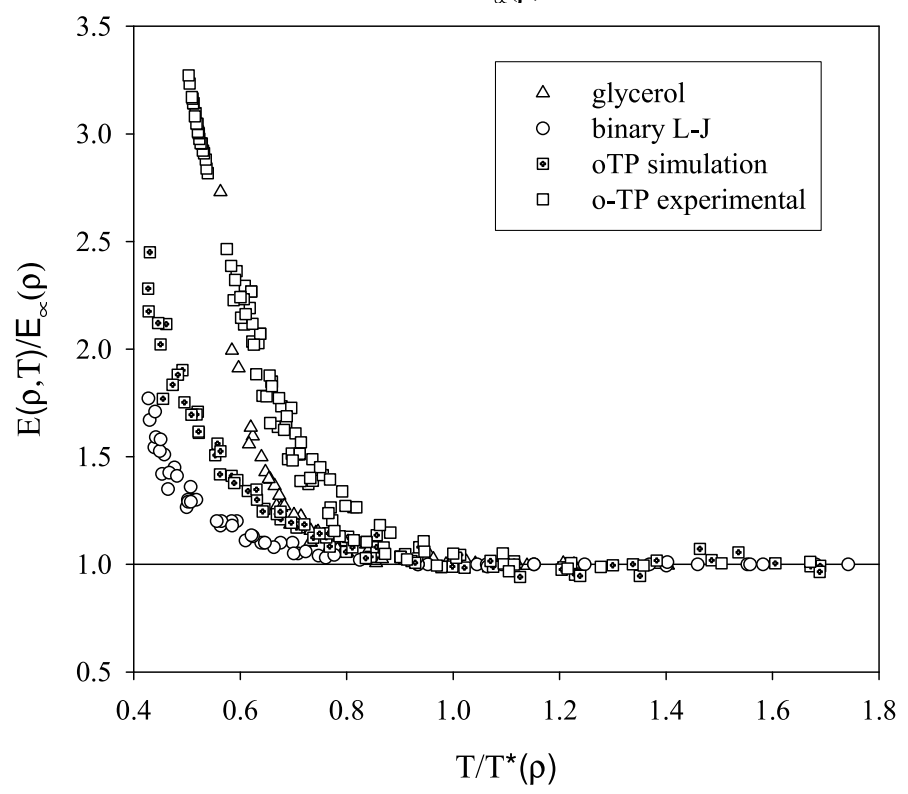

FIG. 8: $E(\rho, T) / E_{\infty}(\rho)$ versus (Top) $\ln \left(T / E_{\infty}(\rho)\right)$ and (Bottom) $T / T^{*}(\rho)$ for the 4 glassformers indicated in the text and in Fig. [7 $T^{*}(\rho)=\zeta_{*} E_{\infty}(\rho)$ is the crossover temperature above which the $T$-dependence of $\eta$ or $\tau_{\alpha}$ is essentially Arrhenius-like. $\zeta_{*}$ is species-specific: $\zeta_{*}=0.42$ for the binary Lennard-Jones mixture; $\zeta_{*}=0.075$ for glycerol; $\zeta_{*}=0.53$ for the model ortho-terphenyl; $\zeta_{*}=17$ for real ortho-terphenyl.

where $\zeta_{g}=T_{g}(\rho) / E_{\infty}(\rho)$ and $d(\Phi(\zeta) / \zeta) / d \zeta$ is negative. According to the above definition of $T_{g}(\rho)$ and to Eq. (6), $\zeta_{g}$ is simply the solution of the equation $\left(\Phi\left(\zeta_{g}\right) / \zeta_{g}\right)=\mathcal{K}-\ln \left(\tau_{\alpha}^{\infty}\right)$, and is thus independent of $\rho$. As a result, whatever the value of $\mathcal{K}$ chosen, the steepness index $m$ is independent of the density. This remains true if the prefactor $\tau_{\alpha}^{\infty}$ has a small residual $\rho$ dependence. The opposite conclusion reached in Ref. [26] from data on the binary Lennard-Jones mixture probably arises from the large intrinsic inaccuracy associated with fitting a 3-parameter Vogel-Fulcher-Tammann formula to a non-fragile system (see Fig. 8) over a limited time-scale domain when the putative $T_{o}$ is at much lower 
temperature than accessible by computer simulation.

The fact that fragility is independent of density does not imply, however, that it is independent of pressure. Fragility measured along isobaric paths involves additional effects, associated with the variation with $\rho$ of $E_{\infty}$ and of the isobaric coefficient of expansivity $\alpha_{P}$. As a result, fragility may increase, decrease, or be nonmonotonic as a function of pressure. We will discuss this point in more detail in a forthcoming publication.

\section{CONCLUSION}

Extending our earlier work we have clarified the respective role of density and temperature in the viscous slowing down of glassforming liquids and polymers. On the basis of available experimental data, we have argued that this slowing down is best described at zeroth-order in terms of thermally activated processes whose characteristic super-Arrhenius behavior is not primarily driven by a congestion or jamming phenomenon resulting, for instance, from a drainage of free volume as one approaches the glass transition, but should rather be explained as an intrinsic temperature effect operating at constant density. Although density effects cannot be neglected at a quantitative level, we have brought additional evidence showing that these effects can be described via a single effective interaction energy $E_{\infty}(\rho)$ that characterizes the relaxation of the liquid in its high-temperature range. In the range of accessible liquid densities, the variation of $E_{\infty}$ with $\rho$ is essentially linear and featureless.

As a result of the collapse of the viscosity and $\alpha$ relaxation time data obtained at various densities onto a mastercurve, we have shown that the (dynamic) fragility of glassforming liquids, i.e., their degree of departure from the high- $T$ Arrhenius behavior, is independent of density. This, in turn, supports the above conclusion that a physical explanation of the super-Arrhenius behavior of the viscosity and $\alpha$-relaxation time as one approaches the glass transition should be found in the intrinsic effect of temperature. Although we feel that we have provided strong evidence to support our conclusions, is should again be stressed that there is a need for more extensive sets of experimental data and accurate equations of state to test on a wider scale the proposed scaling form of the density dependence of the viscosity and the $\alpha$-relaxation time.

\section{Acknowledgments}

LPTL and LPC are UMR 7600 and 8000, respectively, at the CNRS.
[1] M. D. Ediger, C. A. Angell, and S. R. Nagel, J. Phys. Chem. 100, 13200 (1996).

[2] G. Tarjus and D. Kivelson, in "Jamming and Rheology: Constrained Dynamics on Microscopic and Macroscopic Scales", A. Liu and S. R. Nagel Eds. (Taylor and Francis, London, 2001), p. 20.

[3] W. Götze, in "Liquids, Freezing, and the Glass Transition”, J. P. Hansen, D. Levesque, and J. Zinn-Justin Eds. (North Holland, Amsterdam, 1991), p. 287.

[4] D. Kivelson, S. A. Kivelson, X.-L. Zhao, Z. Nussinov, and G. Tarjus, Physica A 219, 27 (1995).

[5] J. H. Gibbs and E. A. Di Marzio, J. Chem. Phys. 28, 373 (1958).

[6] T. R. Kirkpatrick, D. Thirumalai, and P. G. Wolynes, Phys. Rev. A 40, 1045 (1989).

[7] M. Mezard and G. Parisi, Phys. Rev. Lett. 82, 747 (1998).

[8] M. H. Cohen and D. Turnbull, J. Chem. Phys. 31, 1164 (1964); D. Turnbull and M. H. Cohen, ibid. 34, 120 (1961); ibid. 52, 3038 (1970).

[9] A. J. Liu and S. R. Nagel, Nature (London) 396, 21 (1998).

[10] M. Goldstein, J. Chem. Phys. 51, 3728 (1969).

[11] F. H. Stillinger, Science 267, 1935 (1995); P. G. Debenedetti and F. H. Stillinger, Nature (London) 410, 259 (2001).

[12] M. L. Ferrer, C. Lawrence, B. G. Demirjian, D. Kivelson, G. Tarjus, and C. Alba-Simionesco, J. Chem. Phys. 109, 8010 (1998); G. Tarjus, C. Alba-Simionesco, M. L. Ferrer, H. Sakai, and D. Kivelson, in "Slow Dynamics in
Complex Systems", M. Tokuyama and I. Oppenheim Eds. (ACS Books, Washington, 1999), p. 406.

[13] C. Alba-Simionesco, D. Kivelson, and G. Tarjus, J. Chem. Phys. 116, 5033 (2002).

[14] M. Naoki, H. Endou, and K. Matsumoto, J. Chem. Phys. 91, 4169 (1987).

[15] M. Paluch, R. Casalini, and C. M. Roland, Phys. Rev. B 66, 092202 (2002); C. M. Roland and R. Casalini, Macromolecules 36, 1361 (2003).

[16] S. Sastry, PhysChemComm. 14, (2000).

[17] G. P. Johari and E. Whalley, Faraday Symp. Chem. Soc. 6, 23 (1973).

[18] R. L. Cook, H. E. King Jr., C. A. Herbst, and D. R. Herschbach, J. Chem. Phys. 100, 5178 (1994).

[19] A. Tölle, Rep. Prog. Phys. 64, 1473 (2001).

[20] C. Dreyfus, A. Aouadi, J. Gapinski, M. Matos-Lopez, W. Steffen, A. Patkowki, and R. M. Pick, Phys. Rev. E, in press (2003).

[21] S. Mossa, E. La Nave, H. E. Stanley, C. Donati, F. Sciortino, and P. Tartaglia, Phys. Rev. E 65, 041205 (2002).

[22] G. Wahnström and L. J. Lewis, Physica A 201, 150 (1993); L. J. Lewis and G. Wahnström, Phys. Rev. E 50, 3865 (1994).

[23] K. U. Schug, H. E. King, and R. Böhmer, J. Chem. Phys. 109, 1472 (1998).

[24] J. P. Hansen and J . R. Mc Donald, Theory of Simple Liquids, (Academic Press Limited, London, 1986).

[25] C. A. Angell, J. Non-Cryst. Solids 131-133, 13 (1991).

[26] S. Sastry, Nature (London) 409, 164 (2001). 
[27] H. Fujimori, M. Oguni, and C. Alba-Simionesco, Prog. Theor. Phys. 126, 250 (1997).

[28] R. L. Cook, C. A. Herbst, and H. E. King Jr., J. Phys. Chem. 97, 2355 (1993).

[29] A. Chauty-Cailliaux, C. Alba-Simionesco, D. Gomez, and A. Alegria, preprint (2003).

[30] A. Chauty-Cailliaux and C. Alba-Simionesco, private communication (2003).

[31] D. Huang, D. M. Colucci, and G. B. McKenna, J. Chem.
Phys. 116, 3925 (2002).

[32] A. G. S. Hollander and K. O. Pruis, J. Non-Cryst. Solids 286, 1 (2001).

[33] W. T. Laughlin and D. R. Uhlmann, J. Phys. Chem. 76, 2317 (1972); M. Cukierman, J. W. Lane, and D. R. Uhlmann, J. Chem. Phys. 59, 3639 (1973).

[34] R. J. Greet and T. Turnbull, J. Chem. Phys. 46, 1243 (1967). 